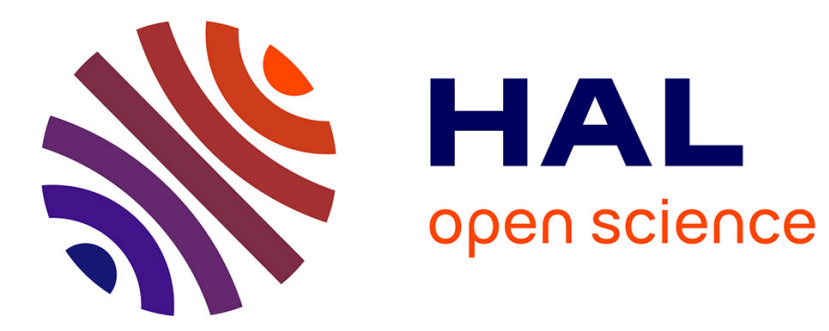

\title{
Emotional Expressions Reinstate Recognition of Other-Race Faces in Infants Following Perceptual Narrowing
}

\author{
Paul C. Quinn, Kang Lee, Olivier Pascalis
}

\section{To cite this version:}

Paul C. Quinn, Kang Lee, Olivier Pascalis. Emotional Expressions Reinstate Recognition of OtherRace Faces in Infants Following Perceptual Narrowing. Developmental Psychology, 2020, 56 (1), pp.15-27. 10.1037/dev0000858 . hal-02567174

\section{HAL Id: hal-02567174 \\ https://hal.science/hal-02567174}

Submitted on 7 May 2020

HAL is a multi-disciplinary open access archive for the deposit and dissemination of scientific research documents, whether they are published or not. The documents may come from teaching and research institutions in France or abroad, or from public or private research centers.
L'archive ouverte pluridisciplinaire $\mathbf{H A L}$, est destinée au dépôt et à la diffusion de documents scientifiques de niveau recherche, publiés ou non, émanant des établissements d'enseignement et de recherche français ou étrangers, des laboratoires publics ou privés. 


\author{
Emotional Expressions Reinstate Recognition of Other-Race Faces \\ in Infants Following Perceptual Narrowing
}

\author{
Paul C. Quinn \\ Kang Lee \\ University of Delaware \\ University of Toronto
}

Olivier Pascalis

Universite Grenoble Alpes

Author Note

Paul C. Quinn, Department of Psychological and Brain Sciences, University of Delaware; Kang Lee, Department of Applied Psychology and Human Development, University of Toronto, Canada; Olivier Pascalis, LPNC, Université Grenoble Alpes, France.

This research was supported by grant R01 HD-46526 from the National Institute of Child Health and Human Development.

Correspondence concerning this article should be addressed to Paul C. Quinn, Department of Psychological and Brain Sciences, University of Delaware, Newark, DE 19716. Email: pquinn@udel.edu 


\begin{abstract}
Perceptual narrowing has been observed in human infants for other-race faces. The hypothesis that adding emotional expressiveness to other-race faces would help infants $(N=128)$ break through narrowing and reinstate other-race face recognition was tested. Experiment 1 demonstrated narrowing for Caucasian infants viewing neutral Asian faces: whereas 3-montholds differentiated Asian faces, 6-month-olds did not. Experiment 2 showed that Caucasian 6month-olds differentiated Asian faces depicted with angry or happy expressions. Experiments 3 and 4 yielded the same results for 6- and 9-month-olds tested with African faces. The outcomes indicate that recognition of other-race faces can be restored with emotional expressiveness. The findings suggest that there is early perceptual-social linkage in face representation that arises in part from a social-to-perceptual pathway.
\end{abstract}




\section{Emotional Expressions Reinstate Recognition of Other-Race Faces \\ in Infants Following Perceptual Narrowing}

Infants are sensitive to differential experience between frequently and infrequently experienced stimuli in their environment (e.g., Hadley, Rost, Fava, \& Scott, 2014). One manifestation of such sensitivity is narrowing, a phenomenon in which the perceptual system of infants becomes tuned to optimally process frequently encountered stimuli while tuning out information that differentiates among less frequently experienced stimuli (Scott, Pascalis, \& Nelson, 2007). Narrowing has been observed across multiple domains, and is in evidence for infant processing of language, music, and face race and species, as well as audio-visual cross-modal processing (for reviews, see Maurer $\&$ Werker, 2014; Pascalis et al., 2014; Watson, Robbins, \& Best, 2014). In the case of face race, infants are initially able to discriminate among own-race faces as well as among other-race faces. However, with increased age accompanied by increased exposure to own-race faces and a lack of exposure to other-race faces, infants maintain the ability to discriminate own-race faces, but have increased difficulty discriminating other-race faces (Kelly et al., 2007, 2009; for a review, see Anzures et al., 2013; and for a recent meta-analysis, see Sugden \& Marquis, 2017).

Given the adaptive advantages of preserving sensitivity to infrequently experienced stimulus inputs (e.g., learning foreign languages, responding to diverse face types without bias), researchers have become interested in discovering the variables that can disrupt such early onset narrowing (e.g., Friendly, Rendall, \& Trainor, 2013). With regard to narrowing in other-race face processing, research shows that exposure to other-race faces for a duration of several weeks or months allows recognition of other-race faces by infants to be maintained or restored. For example, Heron-Delaney et al. (2011) showed that recognition of other-race faces is maintained at 9 months if, starting at 6 months, infants receive experience with photographs of named other-race faces. In addition, 
Anzures et al. (2012) reported that exposure to other-race faces can undo perceptual narrowing for infants who already show it: Between 8 to 10 months, when infants display difficulty recognizing other-race faces, they begin to show above-chance recognition of other-race faces after two to three weeks of daily viewing of videos of named other-race faces.

In the present study, we tested an alternative pathway to restoring infant sensitivity to otherrace faces. In particular, we tested the hypothesis that instead of providing infants with intensive exposure to other-race faces over an extended period of time, adding emotional expressiveness (of either positive or negative valence) to other-race faces can produce an immediate effect of facilitation on other-race face recognition. In other words, this hypothesis suggests that seeing other-race faces with positive or negative emotional expressiveness will break through the narrowing and enhance infant ability to recognize other-race faces. The hypothesis is derived from the broader idea advanced by various authorship groups that there is overlap between perceptual and social processing whereby perceptual processing influences social processing and vice versa (Adams, Ambady, Nakayama, \& Shimojo, 2011; Blandon-Gitlin, Pezdek, Saldivar, \& Steelman, 2014; Freeman, Pauker, \& Sanchez, 2016; Hugenberg, Young, Sacco, \& Bernstein, 2011; Johnson \& Fredrickson, 2005; Lebrecht, Pierce, Tarr, \& Tanaka, 2009; Lee, Quinn, \& Heyman, 2017a; Lee, Quinn, \& Pascalis, 2017b; Quinn, Lee, \& Pascalis, 2018; Todorov, Olivola, Dotsch, \& MendeSiedlecki, 2015; Xiao et al., 2015; Zebrowitz \& Montepare, 2008).

No direct evidence exists to support the hypothesis that facial expression of emotion will interrupt perceptual narrowing because to our knowledge all existing studies of narrowing in face processing have used neutral faces. Nevertheless, this hypothesis is in line with recent advances in research on the interaction between face perception and emotion processing. For decades, it was commonly believed that processing of facial identities and categories (e.g., race) operated 
independently from the processing of facial expressions of emotion (e.g., Bruce \& Young, 1986). While some neural studies have supported this independence hypothesis in adults (e.g., Kubota \& Ito, 2007), behavioral studies have demonstrated interaction of these dimensions. For example, well-known faces have been found to be recognized better by adults when those faces show emotional expression (Gallegos \& Tranel, 2005; Kaufmann \& Schweinberger, 2004). In addition, happy faces have been associated with efficient processing in visual search tasks in adults (Becker, Anderson, Mortenson, Neufeld, \& Neel, 2011). Even more pertinent is the finding that, in adults, changing other-race faces so that they depict angry instead of neutral expressions improves recognition of those faces (Ackerman et al., 2006): Whereas white participants have difficulty recognizing neutral black faces, they have no difficulty doing so when the faces display angry expressions. Moreover, developmental evidence indicates both angry and happy facial expressions facilitate own-race face recognition in infants (Gross \& Schwarzer, 2010; Turati, Montirosso, Brenna, Ferrara, \& Borgatti, 2011).

Based on the adult and developmental data, the present study investigated whether adding angry or happy emotion to Asian or African other-race faces would restore recognition of those faces in Caucasian infants. We specifically focused on infants at an age for which perceptual narrowing had been manifested when the same faces were presented with neutral expressions. Our empirical approach therefore entailed first identifying the age at which infants show difficulty in discriminating other-race faces with neutral expressions. We then sought to determine whether presenting the same faces with an angry or happy expression in this age group would restore recognition of those faces.

We conducted four experiments. In Experiment 1, we tested Caucasian infants with neutral Asian faces to replicate existing findings that 3-month-old Caucasian infants would recognize Asian 
faces, but 6-month-old Caucasian infants would not. We used the visual paired-comparison (VPC) task that has been commonly used for measuring perceptual narrowing in infants (Kelly et al., 2007, 2009). Infants were presented with one Asian face as the familiarization stimulus, and then tested for recognition by presenting the familiarized Asian face paired with a novel Asian face side by side. We used greater looking time to the novel face relative to the familiarized face at test to index face recognition. If infants were able to recognize individual Asian faces, they should look significantly longer at the novel Asian face than the familiarized one (i.e., novelty preference). This experiment aimed to establish the robust narrowing phenomenon of other-race face recognition with our specific infant population, procedure, and set of face stimuli. Experiment 2 used the same procedure as Experiment 1 with 6-month-old Caucasian infants except that we added happy facial expressions (for the Happy condition) or angry facial expressions (for the Angry condition) to the same Asian faces used in Experiment 1. To assess the generalizability of the findings of Experiments 1 and 2, we conducted Experiments 3 and 4 with African faces. In Experiment 3, we tested 6- and 9-month-old Caucasian infants with neutral African faces to establish that 6-month-old Caucasian infants would recognize African faces but 9-month-old Caucasian infants would not. In Experiment 4, we added happy and angry facial expressions to the same African faces used in Experiment 3 for the Happy and Angry conditions, respectively.

\section{General Method}

\section{Participants}

The infants in each experiment were all Caucasian and from predominantly from middleclass backgrounds. All of the parents reported that their infant had little to no experience with Black or Asian faces. The local racial demographics include $87 \%$ White, $6 \%$ Black, and $4 \%$ Asian. 


\section{Stimuli}

The stimuli consisted of photographs of Asian and African neutral, angry, and happy female faces that were taken from the NimStim face set (Tottenham et al., 2009). There were 4 faces for each combination of race and emotion category. The particular stimulus faces used from the Nimstim set were: 15, 16, 17, and 18 (Asian), and 11, 12, 13, and 14 (African). The neutral, angry, and happy facial expressions used were the closed mouth versions. Each photograph was cropped lightly (so as to eliminate hairstyle differences as a differentiating cue), centered, and pasted onto a white 17.7 x $17.7-\mathrm{cm}$ posterboard for presentation. Measuring face height from the top of the forehead to the bottom of the chin and face width at its widest horizontal extent, the neutral, angry, and happy Asian faces had mean heights of $8.40 \mathrm{~cm}(S D=$ $0.72 \mathrm{~cm}), 8.08 \mathrm{~cm}(S D=0.69 \mathrm{~cm})$, and $8.42 \mathrm{~cm}(S D=0.61 \mathrm{~cm})$, and mean widths of $5.83 \mathrm{~cm}$ $(S D=0.32 \mathrm{~cm}), 5.78 \mathrm{~cm}(S D=0.15 \mathrm{~cm})$, and $5.82 \mathrm{~cm}(S D=0.19 \mathrm{~cm})$, respectively. The corresponding measurements for the neutral, angry, and happy African faces had mean heights of $8.43 \mathrm{~cm}(S D=0.59 \mathrm{~cm}), 8.34 \mathrm{~cm}(S D=0.47 \mathrm{~cm})$, and $8.33 \mathrm{~cm}(S D=0.56 \mathrm{~cm})$, and mean widths of $5.53 \mathrm{~cm}(S D=0.28 \mathrm{~cm}), 5.55 \mathrm{~cm}(S D=0.24 \mathrm{~cm})$, and $5.55 \mathrm{~cm}(S D=0.19 \mathrm{~cm})$, respectively. Planned contrasts, conducted on each measure, comparing the means of the happy and angry faces to the mean of the neutral faces for each race, did not yield any significant effects, all $t$ s $<$ $.64, p>.54$, in each instance.

\section{Apparatus}

Infants were tested in a visual preference apparatus, modeled after that of Fagan (1970). The apparatus has a gray display panel that includes two compartments to hold the stimuli. The stimuli were illuminated by a fluorescent lamp that was shielded from the infant's view. Centerto-center distance between compartments was $30.5 \mathrm{~cm}$ and on all trials the display panel was 
situated approximately $30.5 \mathrm{~cm}$ in front of the infant. There was a $0.62 \mathrm{~cm}$ peephole located midway between the compartments that permitted an observer to record infant visual fixations. A second peephole, $0.90 \mathrm{~cm}$ in diameter, located directly below the first peephole, permitted a Pro Video CVC-120PH pinhole camera and Magnavox DVD recorder to record infant gaze duration.

\section{Procedure}

All infants underwent the following general procedure. The infant was brought to the laboratory by a parent and seated in a reclining position on the parent's lap. There were two experimenters, both of whom were naive to the hypotheses under investigation. The first experimenter positioned the apparatus so that the midline of the infant's head was aligned with the midline of the display panel. On each familiarization trial, the first experimenter loaded the stimuli into the compartments of the display panel, and closed the panel, thereby exposing the stimuli to the infant. The parent was unable to see the stimuli.

During each familiarization trial, the first experimenter observed the infant through the small peephole and recorded visual fixations to the left and right stimuli by means of two 605 XE Accusplit electronic stopwatches (San Jose, CA, USA), one of which was held in each hand. The second experimenter timed the duration of the familiarization trials and signaled the end of each trial. Between familiarization trials, the first experimenter opened the panel, recorded infant looking times, and reclosed the panel. The first and second experimenters changed places for the test trials. The experimenter who presented the stimuli and measured infant fixations during familiarization now measured trial duration and signaled the end of each test trial, whereas the second experimenter presented the test stimuli and measured infant fixations. The second experimenter was always naive with respect to the information presented to the infant during familiarization. The two experimenters changed roles across infants. 
Trained observers, naive to the hypotheses, recorded looking times to the stimuli. Interobserver agreement, as determined by comparing looking times measured by the experimenter using the center peephole, and an additional naive observer measuring looking times offline from DVD records, was calculated for the test trials of 32 randomly selected infants participating across the four experiments ( 8 infants from each experiment or $25 \%$ of the infants tested in the four experiments). Average level of agreement for the novel category preference scores was $98.78 \%(S D=1.22)$. In addition, the average of the difference scores for the original and reliability-check novel category preference scores was $0.16, S D=0.95$, which was not significantly different from $0, t(31)=0.95, p=.35$, two-tailed, indicating that the differences between observers were randomly distributed, rather than having one observer with consistently higher or lower scores relative to the other observer.

\section{Initial Control Experiment}

Perceptual narrowing based on face race is well established in the literature (Sugden \& Marquis, 2017). In addition, the focus of the present paper is on the downturn in discrimination of other-race faces and such discrimination may be restored. Nevertheless, because perceptual narrowing implies maintenance of discrimination for own-race faces, we first sought to establish that discrimination of neutral Caucasian faces by Caucasian infants would be maintained in 3-, 6-, and 9-month-olds, using the same testing parameters employed in the four experiments with other-race faces. The particular Caucasian female faces used from the Nimstim set were: 7, 8, 9, and 10. The faces had a mean height of $8.42 \mathrm{~cm}(S D=0.54 \mathrm{~cm})$ and a mean width of $5.66 \mathrm{~cm}$ $(S D=0.30 \mathrm{~cm})$. Each of 163 -month-olds $(10$ females, mean age $=112.56$ days, $S D=13.10$ days), 6-month-olds (9 females, mean age $=189.93$ days, $S D=14.02$ days $)$, and 9-month-olds ( 7 females, mean age $=282.87$ days, $S D=15.52$ days) was familiarized with one Caucasian face 
for 2, 15-s familiarization trials, and then tested with the familiar face paired with a novel Caucasian face for 2, 10-s test trials. Familiarization and test faces were randomly selected for each infant. Left-right positioning of the stimuli was counterbalanced across infants on the first test trial and reversed on the second test trial. Mean novelty preference was above chance for the 3-month-olds $(M=60.17 \%, S D=9.22, t[15]=4.41, p=.0005$, two-tailed, $d=1.10)$, 6-montholds $(M=63.36 \%, S D=10.52, t[15]=5.08, p=.0001$, two-tailed, $d=1.27)$, and 9-month-olds $M=63.51 \%, S D=9.64, t[15]=5.61, p=.00005$, two-tailed, $d=1.40)$, and the effect of age was not significant, $F(2,45)=0.58, p=.56$. The results of the initial control experiment demonstrate that the infants can differentiate among own-race Caucasian faces throughout the developmental period investigated in Experiments 1-4.

\section{Experiment 1}

A VPC procedure was used to establish evidence for perceptual narrowing in Caucasian 3- to 6-month-olds viewing neutral other-race Asian faces. Each infant was familiarized with one neutral Asian female face for 2, 10-s familiarization trials, and then tested with the familiar exemplar paired with a novel neutral Asian female face for 2, 10-s test trials. Discrimination was inferred if infants showed an above-chance preference for the novel exemplars. Because prior studies reported above-chance discrimination of neutral Asian faces by Caucasian 3-month-olds (Kelly et al., 2007), but chance-level discrimination of those faces by Caucasian 6-month-olds (Anzures, Quinn, Pascalis, Slater, \& Lee, 2010; Anzures et al., 2011; Quinn, Lee, Pascalis, \& Tanaka, 2016), the expectation was for the 3-month-olds, but not the 6-month-olds, to display above-chance discrimination performance.

\section{Method}

Participants. Participants were 16 Caucasian 3-month-olds (7 females), mean age = 
109.81 days, $S D=12.67$ days, and 16 Caucasian 6-month-olds (9 females), mean age $=192.69$ days, $S D=11.25$ days. One additional 3-month-old was tested but excluded from the data analysis because of failure to compare the test stimuli; one additional 6-month-old was tested, but did not complete the procedure due to fussiness.

Stimuli. Stimuli were the neutral Asian faces described in the General Method section.

Procedure. Each infant from both age groups was familiarized with one neutral Asian face for 2, 15-s familiarization trials, and then tested with the familiar face paired with a novel neutral Asian face for 2, 10-s test trials. Familiarization and test faces were randomly selected for each infant in the 3-month-old group and those same familiarization and test faces were presented to the infants in the 6-month-old group. Left-right positioning of the stimuli was counterbalanced across infants on the first test trial and reversed on the second test trial.

\section{Results and Discussion}

Familiarization Trials. Individual looking times were summed over left and right copies of the stimulus presented on each trial. Mean looking times on Trial 1 and on Trial 2 for the two age groups are shown in Table 1. An analysis of variance (ANOVA), Age (3 months vs. 6 months) $\mathrm{x}$ Trials (1 vs. 2), performed on the individual scores revealed only a significant Trials effect, $F(1,30)=4.33, p=.046$, partial eta ${ }^{2}=.13$. Neither the effect of Age, $F(1,30)=.530, p=$ .472 , nor the interaction of Trials $x$ Age, $F(1,30)=.100, p=.754$, were significant. Using the standard operational definition of habituation as a decline in responsiveness with repeated stimulation (Cohen \& Gelber, 1975), the decrement in looking time from the first to the second trial of familiarization supports the argument that both age groups habituated to the stimuli.

Preference Test Trials. Mean novelty preference scores are shown in Table 1 and also depicted in Figure 1. While 3-month-olds displayed above-chance discrimination of the faces, 6- 
month-old performance did not differ from chance. Examined from the perspective of individual infants, at 3 months, 15 of 16 infants displayed novelty preference scores above $50 \%, p=.0005$, whereas at 6 months, only 9 of 16 infants did so, $p=.804$. The findings provide evidence of perceptual narrowing for Asian faces among Caucasian infants between 3 and 6 months.

\section{Experiment 2}

Experiment 2 replicated Experiment 1, but with angry or happy faces being shown during both the familiarization and preference test (instead of neutral faces), and only with 6-montholds, the post-narrowing age group. Experiment 2 was performed to determine if adding angry or happy expressions to other-race faces would allow infants to recognize them. The addition of angry expressions was undertaken because adult work has shown that changing other-race faces from neutral to angry releases adult perceivers from the other-race effect and restores recognition of those faces (Ackerman et al., 2006), and infant work has shown that face recognition is enhanced for faces when those faces are presented as angry rather than as neutral (Gross \& Schwarzer, 2010). The addition of happy expressions was undertaken because of evidence in adults that happy facial expressions can be detected more efficiently than some negative facial expressions, including anger (Becker et al., 2011) and sadness (Srivastava \& Srinivasan, 2010), and evidence in infants that happy expressions can enhance recognition relative to neutral expressions (Turati et al., 2011).

\section{Method}

Participants. Participants were 32 Caucasian 6-month-olds (17 females), mean age = 192.72 days, $S D=13.49$ days. One additional infant was tested, but did not complete the procedure due to fussiness. 
Stimuli. Stimuli were the angry and happy Asian faces described in the General Method section.

Procedure. Procedure was the same as in Experiment 1, except that only 6-month-olds were tested, and happy or angry expressions replaced neutral expressions during both familiarization and preference test. Half of the infants were familiarized and tested with angry faces, the other half with happy faces. The same randomly selected pairings of faces used in Experiment 1 were used in Experiment 2 so that the addition of emotion to the faces would not be confounded with the processing of the identity of the faces.

\section{Results and Discussion}

Familiarization Trials. Mean looking times are shown in Table 1. An ANOVA, Facial Expression (Angry vs. Happy) x Trials (1 vs. 2), performed on the individual scores revealed only a significant Trials effect, $F(1,30)=6.35, p=.017$, partial eta ${ }^{2}=.17$. Neither the effect of Facial Expression, $F(1,30)=1.31, p=.261$, nor the interaction of Face Category $\mathrm{x}$ Trials, $F(1$, $30)=1.56, p=.221$, were significant. The decrement in looking time from the first to the second trial of familiarization suggests that the 6-month-olds in Experiment 2, like the 6-month-olds in Experiment 1, habituated to the faces, even though they depicted angry or happy (rather than neutral) expressions.

Preference Test Trials. Mean novelty preference scores are shown in Table 1 and also illustrated in Figure 1. $t$ tests comparing the preference scores to $50 \%$ revealed that both groups of infants (i.e., those familiarized with angry and those familiarized with happy faces) preferred to look at the novel stimuli. Moreover, when the mean novelty preference score for infants presented with angry faces was compared with the mean novelty preference score for infants familiarized with happy faces, the difference was not significant, $t(30)=0.92, p=.365$. Analysis 
of individual performance revealed that 13 of 16 infants displayed novelty preference scores above $50 \%$ when presented with either angry or happy facial expressions (binomial probability, $p$ $=.021$, in each case).

In contrast to the chance performance of the 6-month-old Caucasian infants with the neutral Asian faces in Experiment 1, the results of Experiment 2 show that presenting Asian faces with an angry or happy facial expression yields above-chance recognition of those faces in same-aged Caucasian infants. The findings suggest that adding emotion to other-race faces, irrespective of whether that emotion is negative or positive, can restore recognition of those faces following perceptual narrowing.

\section{Experiment 3}

Although Experiments 1 and 2 demonstrated that adding emotion to Asian faces for reinstates recognition of those faces in Caucasian 6-month-olds (the post-narrowing age group for Asian faces), they leave open the question of whether the effect of emotion will extend to classes of other-race faces beyond Asian. Experiment 3 thus began the inquiry of whether the effect of emotion would generalize to African faces. As was the case for Experiment 1, we first had to establish evidence for perceptual narrowing in Caucasian infants, in this case, for neutral African faces. However, in Experiment 3, because prior evidence using the same procedure and stimuli had yielded positive evidence for discrimination of neutral African faces at 6 months (Quinn et al., 2016), we used the VPC procedure of Experiment 1 to assess perceptual narrowing in Caucasian 6- to 9-month-olds viewing neutral other-race African faces. Given the abovechance discrimination of neutral African faces by Caucasian 6-month-olds in Quinn et al. (2016), but chance-level discrimination of neutral African faces by Caucasian 9-month-olds (Kelly et al., 
2007), the expectation was for the 6-month-olds, but not the 9-month-olds, to display abovechance discrimination performance.

\section{Method}

Participants. Participants were 16 Caucasian 6-month-olds (10 females), mean age = 196.19 days, $S D=15.82$ days, and 16 Caucasian 9-month-olds (10 females $)$, mean age $=276.50$ days, $S D=15.59$ days. One additional 6-month-old was tested, but did not complete the procedure due to fussiness.

Stimuli. Stimuli were the neutral African faces described in the General Method section.

Procedure. Procedure was the same as in Experiment 1, except that neutral African faces were presented instead of neutral Asian faces.

\section{Results and Discussion}

Familiarization Trials. Mean looking times are shown in Table 2. An ANOVA, Age (6 months vs. 9 months) x Trials (1 vs. 2), performed on the individual scores revealed only a significant Trials effect, $F(1,30)=4.18, p=.049$, partial eta $^{2}=.12$. Neither the effect of Age, $F(1,30)=.630, p=.434$, nor the interaction of Age x Trials, $F(1,30)=.260, p=.614$, were significant. The decrement in looking time from the first to the second trial of familiarization for both the 6- and 9-month-old infants presented with neutral African faces was once more consistent with habituation.

Preference Test Trials. Mean novel category preference scores are shown in Table 2 and also depicted in Figure 2. While 6-month-olds displayed above-chance recognition, 9-month-old performance did not differ from chance. Examined from the perspective of individual infants, at 6 months, 13 of 16 infants displayed novelty preference scores above $50 \%, p=.021$, whereas at 
9 months, only 9 of 16 infants did so, $p=.804$. The findings provide evidence of perceptual narrowing for neutral African faces among Caucasian infants between 6 and 9 months.

\section{Experiment 4}

Experiment 4 replicated Experiment 2, but with angry or happy African faces instead of angry or happy Asian faces, and with Caucasian 9-month-olds rather than Caucasian 6-montholds. It was designed to determine whether the facilitative effect of angry and happy expressions on recognition of Asian faces that was observed in Experiment 2 for Caucasian 6-month-olds would also be observed for African faces in Caucasian 9-month-olds.

\section{Method}

Participants. Participants were 32 Caucasian 9-month-olds (14 females), mean age = 275.63 days, $S D=13.36$ days. One additional infant was tested, but did not complete the procedure due to fussiness.

Stimuli. Stimuli were the angry and happy African faces described in the General Method section.

Procedure. Procedure was the same as in Experiment 2, except that 9-month-olds were tested, and angry or happy expressions replaced neutral expressions during both familiarization and preference test. The same randomly selected pairings of faces used in Experiment 3 were used in Experiment 4 so that the addition of emotion to the faces was not confounded with the processing of face identity.

\section{Results and Discussion}

Familiarization Trials. Mean looking times are shown in Table 2. An ANOVA, Facial Expression (Angry vs. Happy) x Trials (1 vs. 2), performed on the individual scores revealed only a significant Trials effect, $F(1,30)=13.17, p=.001$, partial eta ${ }^{2}=.30$. Neither the effect of 
Facial Expression, $F(1,30)=0.00, p=1.00$, nor the interaction of Face Category $\mathrm{x}$ Trials, $F(1$, $30)=0.68, p=.416$, were significant. The decrement in looking time from the first to the second trial of familiarization suggests that the 9-month-olds in Experiment 4, like the 6-month-olds in Experiment 2, habituated to the angry and happy faces, even though they were from a different other-race class.

Preference Test Trials. Mean novelty preference scores are shown in Table 2 and also illustrated in Figure 2. $t$ tests comparing the preference scores to $50 \%$ revealed that both the infants familiarized with angry expressions and those familiarized with happy expressions preferred to look at the novel stimuli. Moreover, when the mean novelty preference score for infants presented with angry expressions was compared with the mean novelty preference score for infants familiarized with happy expressions, the difference was not significant, $t(30)=0.66, p$ $=.51$. Analysis of individual performance revealed that 14 of 16 infants displayed novelty preference scores above $50 \%$ when presented infants were presented with either angry or happy expressions (binomial probability, $p=.004$, in each case).

Whereas the 9-month-old Caucasian infants presented with neutral African faces displayed chance recognition performance in Experiment 3, the results of Experiment 4 indicate that presenting African faces with an angry or happy facial expression yields above-chance recognition of those faces in same-aged Caucasian infants. The findings from Experiment 4 thus converge with those from Experiment 2 to suggest that adding either positive or negative emotion to other-race faces can restore recognition and eradicate the perceptual narrowing for those faces that had been observed with neutral expressions in Experiments 1 and 3 . 


\section{General Discussion}

The present study tested the hypothesis that facial emotional expressiveness of either negative or positive valence could break through perceptual narrowing and facilitate infant recognition of other-race faces. We found that when presented with other-race faces with a neutral expression, infants displayed the perceptual narrowing phenomenon, whereby younger but not older infants recognized other-race faces (with younger and older infants alike recognizing own-race faces in the initial control experiment). This phenomenon has been reported in prior papers (e.g., Kelly et al., 2007, 2009). Perceptual narrowing was observed from 3 to 6 months for the neutral Asian faces in Experiment 1 and from 6 to 9 months for the neutral African faces in Experiment 3. However, when the other-race faces were presented with either a negative (angry) or positive (happy) expression, perceptual narrowing was not observed. More specifically, 6-month-old Caucasian infants were able to recognize angry and happy Asian faces in Experiment 2, and 9-month-old Caucasian infants were able to recognize angry and happy African faces in Experiment 4.

The present findings support the hypothesis that processing of own- and other-race faces at the category or identity level is influenced by emotional expressiveness (neutral or valenced), at least in older infants. The data are consistent with the view that there is an interaction between the processing of faces of different races and that of emotional expression (e.g., Ackerman et al., 2006). Our findings are inconsistent with the view that the processing of facial identity and category information (i.e., race) is independent of that of emotional expression (e.g., Bruce \& Young, 1986; Kubota \& Ito, 2007). Our data are also interesting in light of Vogel, Monesson, and Scott's (2012) findings that 9-month-olds, but not 5-month-olds, are more sensitive to facesound emotional congruency from own-race face-voice pairs than from other-race face-voice 
pairs, and additional studies reporting an own-race advantage in the recognition of facial expressions of emotion by children and adults (Kilbride \& Yarczower, 1983; Markham \& Wang, 1996; Elfenbein \& Ambady, 2002). The prior outcomes indicate that the processing of face race can affect the processing of emotional information. Our findings indicate that the reverse is true as well. Thus, the current and prior results taken together suggest a bidirectional relation between the processing of emotion and that of face race: changing emotion can affect how race is processed and changing race can affect how emotion is processed. However, the nature of the effect may be different in the two cases. Whereas changing emotion from neutral to valenced (either negative or positive) facilitated recognition of other-race faces in the current study, changing race from own- to other in prior studies impeded recognition of emotion.

A key question concerns the mechanism by which expressions facilitate recognition of other-race faces by infants. One possible mechanism is salience: the faces with emotional expression may be more salient than the faces with neutral expression, resulting in more looking time and a greater likelihood of processing the face identity information. However, in Experiments 1 and 2, the average looking time across the two familiarization trials was 9.38 (SD $=2.85), 7.69(S D=2.90)$, and $8.88(S D=2.98)$ for the 6-month-olds presented with neutral, angry, and happy Asian faces, respectively, with a null effect of emotion, $F(2,45)=1.41, p=$ .255. Likewise, in Experiments 3 and 4, the average looking time across the two familiarization trials was $7.67(S D=3.55), 7.56(S D=2.00)$, and $7.57(S D=2.42)$ for the 9-month-olds presented with neutral, angry, and happy African faces, respectively, with another null effect of emotion, $F(2,45)=0.01, \mathrm{p}=.990$. The equivalent looking times infants displayed during familiarization for the neutral, angry, and happy faces for both races thus do not provide support for differential salience. 
A second possible mechanism is discriminability: adding emotion creates more discriminable stimuli. However, this possibility is contradicted by the equivalent pairwise stimulus similarity ratings obtained from 36 Caucasian adults (age $=35.83$ years, $S D=15.50,21$ females), 12 per condition, on a 1 to 5 Likert scale $(1=$ not at all similar, $5=$ very similar $)$ for the neutral $(M=2.17, \mathrm{SD}=.53)$, angry $(M=2.19, S D=.53)$, and happy $(M=2.21, \mathrm{SD}=.40)$ Asian faces, $F(2,33)=.02, p=.98$. It is likewise refuted by the equivalent pairwise stimulus similarity ratings obtained from a separate group of 36 Caucasian adults (age $=34.36, S D=15.16,20$ females), 12 per condition, using the same Likert scale for the neutral $(M=2.24, \mathrm{SD}=.61)$, angry $(M=2.46, S D=.38)$, and happy $(M=2.54, \mathrm{SD}=.43)$ African faces, $F(2,33)=1.26, p=$ .30. Thus, the similarity ratings suggest that the addition of the emotional expressions to the stimuli did not give rise to stimuli that were perceived as more dissimilar from one another.

Given the lack of support for these two lower-level mechanisms, we are intrigued by the possibility that a higher-level mechanism is at work: infants may be processing the meaning or communicative intention of the angry and happy expressions (Frith, 2009; Senju \& Csibra, 2008). If so, the identity of the angry faces may be processed because such faces pose threat (Ackerman et al., 2006), and the identity of the happy faces may be processed because such faces offer possibility for affiliation (Becker et al., 2011). As noted by Hugenberg, Young, Sacco, and Bernstein (2011), "facial expressions...can create the motive to individuate cross-race faces" (p. 252). From this perspective, it may be that when a face poses threat or invites affiliation, infants are motivated to encode the identity of the face, thus disrupting perceptual narrowing for face race.

The present findings also have important theoretical implications for understanding the relation between perceptual and social information processing and their ontogenesis. Lee, Quinn, 
and colleagues (Lee et al., 2017a, 2017b) have recently proposed a Perceptual-Social Linkage Hypothesis that posits a strong connection between the development of perceptual information processing about faces and that of social information processing about faces. They suggested that due to the asymmetrical exposure to own- versus other-race faces during the initial months of life (greater experience with own- than other-race faces), infants show perceptual processing differences for own- versus other-race faces that in turn lead to down-stream social consequences such as racial biases in early childhood (Quinn et al., 2013, 2016; Xiao et al., 2015). The present findings suggest that the perceptual to social pathway may be an incomplete account of how the relation between perceptual and social processing of faces emerges. Rather than the linkage arising from just a single direction, the perceptual-social linkage may arise bidirectionally: development of perceptual processing of faces affects that of social processing of faces, and vice versa. The current and existing findings taken together thus suggest that early perceptual-social linkage in the representation of faces may come about through multiple pathways. 


\section{References}

Ackerman, J. M., Shapiro, J. R., Neuberg, S. L., Kenrick, D. T., Becker, D. V., Griskevicius, V.,...Schaller, M. (2006). They all look the same to me (unless they're angry): From outgroup homogeneity to outgroup heterogeneity. Psychological Science, 17, 836-840.

Adams, R. B., Jr., Ambady, N., Nakayama, K., \& Shimojo, S. (Eds.). (2011). The science of social vision. New York: Oxford University Press.

Anzures, G., Pascalis, O., Quinn, P. C., Slater, A. M., \& Lee, K. (2011). Minimizing skin color differences does not eliminate the own-race recognition advantage in infants. Infancy, 16, 640-654.

Anzures, G., Quinn, P. C., Pascalis, O., Slater, A. M., \& Lee, K. (2010). Categorization, categorical perception, and asymmetry in infants' representation of face race. Developmental Science, 13, 553-564.

Anzures, G., Quinn, P. C., Pascalis, O., Slater, A. M., Tanaka, J. W., \& Lee, K. (2013). Developmental origins of the other-race effect. Current Directions in Psychological Science, 22, 173-178.

Anzures, G., Wheeler, A., Quinn, P. C., Pascalis, O., Slater, A. M., Heron-Delaney, M., Tanaka, J. W., \& Lee, K. (2012). Brief daily exposures to Asian females reverses perceptual narrowing for Asian faces in Caucasian infants. Journal of Experimental Child Psychology, 112, 484-495.

Becker, D. V., Anderson, U. S., Mortensen, C. R., Neufeld, S. L., \& Neel, R. (2011). The face in the crowd effect unconfounded: happy faces, not angry faces, are more efficiently detected in single- and multiple-target visual search tasks. Journal of Experimental Psychology: General, 140, 637-659. 
Blandon-Gitlin, I., Pezdek, K., Saldivar, S., \& Steelman, E. (2014). Oxytocin eliminates the own-race bias in face recognition memory. Brain Research, 1580, 180-187.

Bruce, V., \& Young, A. (1986). Understanding face recognition. British Journal of Psychology, $77,305-327$.

Cohen, L. B., \& Gelber, E. R. (1975). Infant visual memory. In L. B. Cohen \& P. Salapatek (Eds.), Infant perception: From sensation to cognition (Vol. 1, pp. 347-403). New York, NY: Academic Press.

Elfenbein, H. A., \& Ambady, N. (2002). On the universality and cultural specificity of emotion recognition: A meta-analysis. Psychological Bulletin, 128, 203-235.

Fagan, J. F. (1970). Memory in the infant. Journal of Experimental Child Psychology, 9, $217-$ 226.

Freeman, J. B., Pauker, K., \& Sanchez, D. (2016). A perceptual pathway to bias: Interracial exposure reduces abrupt shifts in real-time race perception that predict mixed-race bias. Psychological Science, 27, 502-517.

Friendly, R. H., Rendall, D., \& Trainor, L. J. (2013). Plasticity after perceptual narrowing for voice perception: reinstating the ability to discriminate to discriminate monkeys by their voices at 12 months of age. Frontiers in Psychology, 4. http://dx.doi.org/10.3389/fpsyg.2013.00718

Frith, C. (2009). Role of facial expressions in social interactions. Philosophical Transactions of the Royal Society B: Biological Sciences, 364, 3453-3458.

Gallegos, D. R., \& Tranel, D. (2005). Positive facial affect facilitates the identification of famous faces. Brain \& Language, 93, 338-348. 
Gross, C., \& Schwarzer, G. (2010). Face recognition across varying poses in seven- and ninemonth-old infants: The role of facial expression. International Journal of Behavioural Development, 34, 417-426.

Hadley, H., Rost, G. C., Fava, E., \& Scott, L. S. (2014). A mechanistic approach to cross-domain perceptual narrowing in the first year of life. Brain Sciences, 4, 613-634.

Heron-Delaney, M., Anzures, G., Herbert, J. S., Quinn, P. C., Slater, A. M., Tanaka, J. W., Lee, K., \& Pascalis, O. (2011). Perceptual training prevents the emergence of the other race effect during infancy. PLoS ONE, 6(5): e19858. doi:10.1371/journal.pone.0019858

Hugenberg, K., Young, S., Bernstein, M., \& Sacco, D. F. (2011). Social categorization influences face perception and face memory. In A. J. Calder, G. Rhodes, M. H. Johnson, \& J. V. Haxby (Eds)., The Oxford handbook of face perception (pp. 753-778). Oxford, UK: Oxford University Press.

Johnson, K. J., \& Fredrickson, B. L. (2005). We all look the same to me: Positive emotions eliminate the own-race bias. Psychological Science, 16, 875-881.

Kaufmann, J. M., \& Schweinberger, S. R. (2004). Expression influences the recognition of familiar faces. Perception, 33, 399-408.

Kelly, D. J., Liu, S., Lee, K., Quinn, P. C., Pascalis, O., Slater, A. M., \& Ge, L. (2009). Development of the other-race effect in infancy: Evidence towards universality? Journal of Experimental Child Psychology, 104, 105-114.

Kelly, D. J., Quinn, P. C., Slater, A. M., Lee, K., Ge, L., \& Pascalis, O. (2007). The other-race effect develops during infancy: Evidence of perceptual narrowing. Psychological Science, 18, 1084-1089. 
Kilbride, J., \& Yarczower, M. (1983). Ethnic bias in the recognition of racial expressions. Journal of Nonverbal Behavior, 8, 27-41.

Kubota, J. T., \& Ito, T. A. (2007). Multiple cues in social perception: The time course of processing race and facial expression. Journal of Experimental Social Psychology, 43, 738-752.

Lebrecht, S., Pierce, L. J., Tarr, M. J., \& Tanaka, J. W. (2009). Perceptual other-race training reduces implicit racial bias. PloS ONE, 4, e4215. doi: 10.1371/journal.pone.0004215

Lee, K., Quinn, P. C., \& Heyman, G. D. (2017). Rethinking the emergence and development of implicit racial bias: A perceptual-social linkage hypothesis. In E. Turiel, N. Budwig, \& P. Zelazo (Eds.), New perspectives on human development (pp. 27-46). Cambridge, UK: Cambridge University Press.

Lee, K., Quinn, P. C., \& Pascalis, O. (2017). Face race processing and racial bias in early development: A perceptual-social linkage. Current Directions in Psychological Science, $26,256-262$.

Markham, R., \& Wang, L. (1996). Recognition of emotion by Chinese and Australian children. Journal of Cross-Cultural Psychology, 27, 616-643.

Maurer, D., \& Werker, J. F. (2014). Perceptual narrowing during infancy: A comparison of language and faces. Developmental Psychobiology (Special Issue on Perceptual Narrowing), 56, 154-178.

Pascalis, O., Loevenbruck, H., Quinn, P. C., Kandel, S., Tanaka, J. W., \& Lee, K. (2014). On the linkage between face processing, language processing, and narrowing during development. Child Development Perspectives, 8, 65-70. 
Quinn, P. C., Anzures, G., Lee, K., Pascalis, O., Slater, A., \& Tanaka, J. W. (2013). On the developmental origins of differential responding to social category information. In M. R. Banaji \& S. A. Gelman (Eds.), Navigating the social world: What infants, children, and other species can teach us (pp. 286-291). New York: Oxford University Press.

Quinn, P. C., Lee, K., \& Pascalis, O. (2018). Perception of face race by infants: Five developmental changes. Child Development Perspectives. https://doi.org/10.1111/cdep.12286

Quinn, P. C., Lee, K., Pascalis, O., \& Tanaka, J. W. (2016). Narrowing in categorical responding to other-race face classes by infants. Developmental Science, 19, 362-371.

Scott, L. S., Pascalis, O., \& Nelson, C. A. (2007). A domain-general theory of the development of perceptual discrimination. Current Directions in Psychological Science, 16, 197-201.

Senju, A. \& Csibra, G. (2008). Gaze following in human infants depends on communicative signals. Current Biology, 18, 668-671.

Srivastava, P., \& Srinivasan, N. (2010). Time course of visual attention with emotional faces. Attention, Perception, \& Psychophysics, 72, 369-377.

Sugden, N. A., \& Marquis, A. R. (2017). Meta-analytic review of the development of face discrimination in infancy: Face race, face gender, infant age, and methodology moderate face discrimination. Psychological Bulletin, 143, 1201-1244.

Todorov, A., Olivola, C. Y., Dotsch, R., \& Mende-Siedlecki, P. (2015). Social attributions from faces: Determinants, consequences, accuracy, and functional significance. Annual Review of Psychology, 66, 519-545. 
Tottenham, N., Tanaka, J. W., Leon, A. C., McCarry, T., Nurse, M., Hare, T. A., ... Nelson, C. A. (2009). The NimStim set of facial expressions: Judgments from untrained research participants. Psychiatry Research, 168, 242-249.

Turati, C., Montirosso, R., Brenna, V., Ferrara, V., \& Borgatti, R. (2011). A smile enhances 3month-olds' recognition of an individual face. Infancy, 16, 306-317.

Vogel, M., Monesson, A., \& Scott, L. S. (2012). Building biases in infancy: the influence of race on face and voice emotion matching. Developmental Science, 15, 359-372.

Watson, T. L., Robbins, R. A., \& Best, C. T. (2014). Infant perceptual development for faces and spoken words: An integrated approach. Developmental Psychobiology, 56, 1454-1481.

Xiao, W. S., Fu, G., Quinn, P. C., Qin, J., Tanaka, J., Pascalis, O., \& Lee, K. (2015).

Individuation training with other-race faces reduces preschoolers' implicit racial bias: A link between perceptual and social representation of faces in children. Developmental Science, 18, 655-663.

Zebrowitz, L. A., \& Montepare, J. M. (2008). Social psychological face perception: Why appearance matters. Social and Personality Psychology Compass, 2, 1497-1517. 
Table 1

Mean Fixation Times (s) During the Familiarization Trials 1-2 and Mean Novelty Preference Scores (\%) During the Test Trials of Experiment 1-2

\begin{tabular}{|c|c|c|c|c|c|c|c|c|c|}
\hline \multirow[b]{3}{*}{ Age, Race, Emo (Exp) } & \multicolumn{4}{|c|}{ Familiarization Fixation Time } & \multicolumn{5}{|c|}{ Novelty Preference } \\
\hline & \multicolumn{2}{|c|}{ Trial 1} & \multicolumn{2}{|c|}{ Trial 2} & \multirow[b]{2}{*}{$M$} & \multirow[b]{2}{*}{$(S D)$} & \multirow[b]{2}{*}{$t^{\mathrm{a}}$} & \multirow[b]{2}{*}{$p$} & \multirow[b]{2}{*}{$d$} \\
\hline & $M$ & $(S D)$ & $M$ & $(S D)$ & & & & & \\
\hline 3 mths, Asi, neu (E1) & 10.79 & $(3.62)$ & 9.42 & $(3.24)$ & 58.23 & $(9.83)$ & 3.35 & .004 & 0.84 \\
\hline 6 mths, Asi, neu (E1) & 9.88 & $(3.09)$ & 8.87 & $(3.07)$ & 51.43 & $(19.03)$ & 0.30 & .768 & 0.08 \\
\hline 6 mths, Asi, ang (E2) & 8.02 & $(2.26)$ & 7.36 & $(4.30)$ & 64.07 & $(16.11)$ & 3.49 & .003 & 0.87 \\
\hline 6 mths, Asi, hap (E2) & 9.86 & $(3.12)$ & 7.90 & $(3.16)$ & 59.24 & $(13.42)$ & 2.75 & .015 & 0.69 \\
\hline
\end{tabular}

${ }^{\mathrm{a}} t$ vs. chance. 
Table 2

Mean Fixation Times (s) During the Familiarization Trials 1-2 and Mean Novelty Preference Scores (\%) During the Test Trials of Experiment 3-4

\begin{tabular}{|c|c|c|c|c|c|c|c|c|c|}
\hline \multirow[b]{3}{*}{ Age, Race, Emo (Exp) } & \multicolumn{4}{|c|}{ Familiarization Fixation Time } & \multicolumn{5}{|c|}{ Novelty Preference } \\
\hline & \multicolumn{2}{|c|}{ Trial 1} & \multicolumn{2}{|c|}{ Trial 2} & \multirow[b]{2}{*}{$M$} & \multirow[b]{2}{*}{$(S D)$} & \multirow[b]{2}{*}{$t^{\mathrm{a}}$} & \multirow[b]{2}{*}{$p$} & \multirow[b]{2}{*}{$d$} \\
\hline & $M$ & $(S D)$ & $M$ & $(S D)$ & & & & & \\
\hline 6 mths, Afr, neu (E3) & 9.06 & $(2.16)$ & 7.98 & $(3.39)$ & 61.91 & $(16.38)$ & 2.91 & .011 & 0.73 \\
\hline 9 mths, Afr, neu (E3) & 7.99 & $(3.73)$ & 7.34 & $(3.61)$ & 49.10 & $(17.31)$ & -0.21 & .806 & -0.05 \\
\hline 9 mths, Afr, ang (E4) & 8.42 & $(2.77)$ & 6.70 & $(2.10)$ & 64.84 & $(11.71)$ & 5.07 & .000 & 1.27 \\
\hline 9 mths, Afr, hap (E4) & 8.92 & $(3.43)$ & 6.21 & $(2.90)$ & 62.13 & $(11.51)$ & 4.22 & .001 & 1.05 \\
\hline
\end{tabular}

${ }^{\mathrm{a}} t$ vs. chance. 


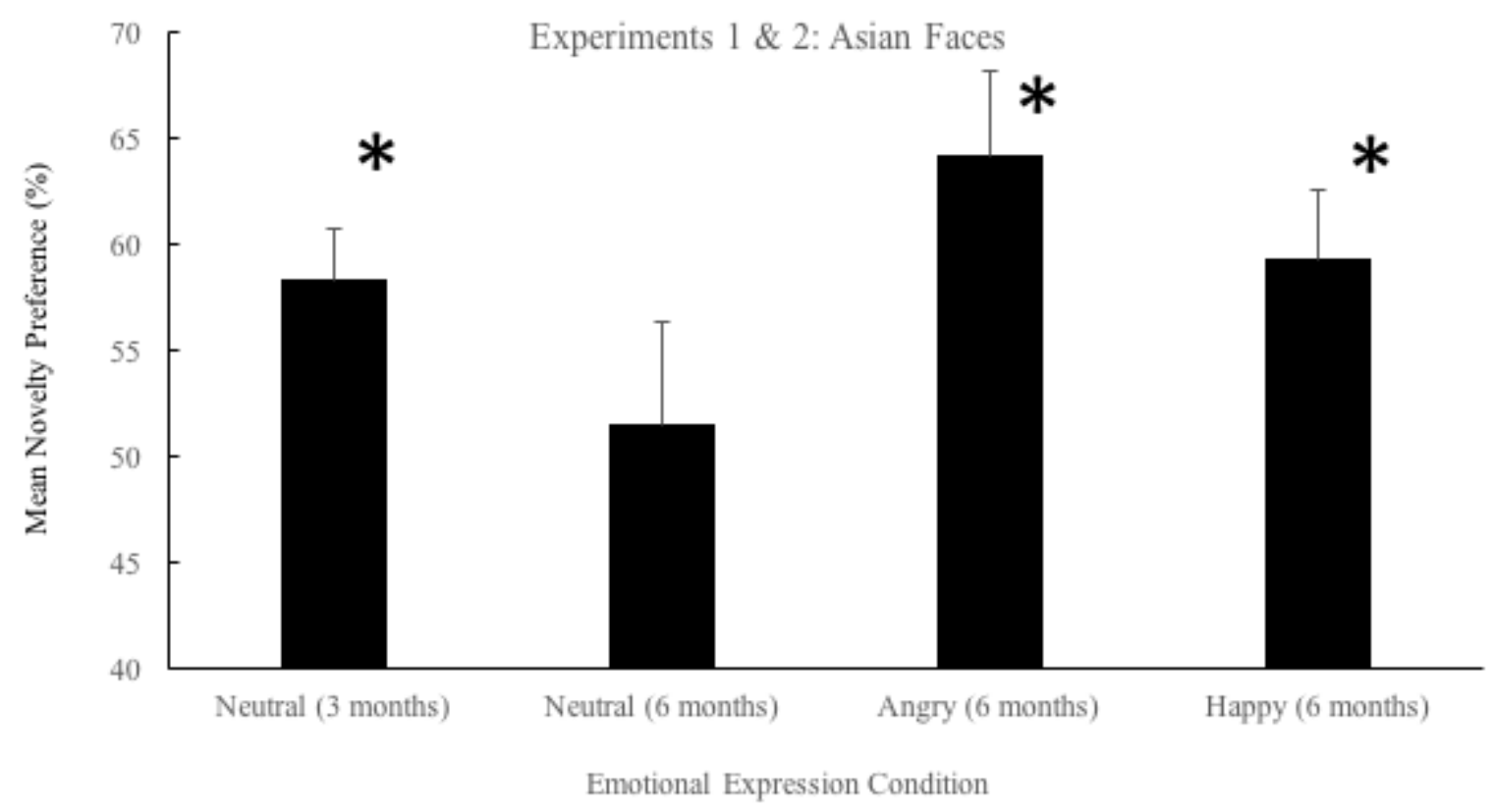

Figure 1. Mean novelty preference scores (in percentages) as a function of the emotional expression condition (neutral, angry, or happy) and the age of the participants (3 or 6 months) in Experiments 1 and 2. Error bars show standard errors of the mean. Scores that differed from chance $(50 \%)$ are marked with an asterisk. 


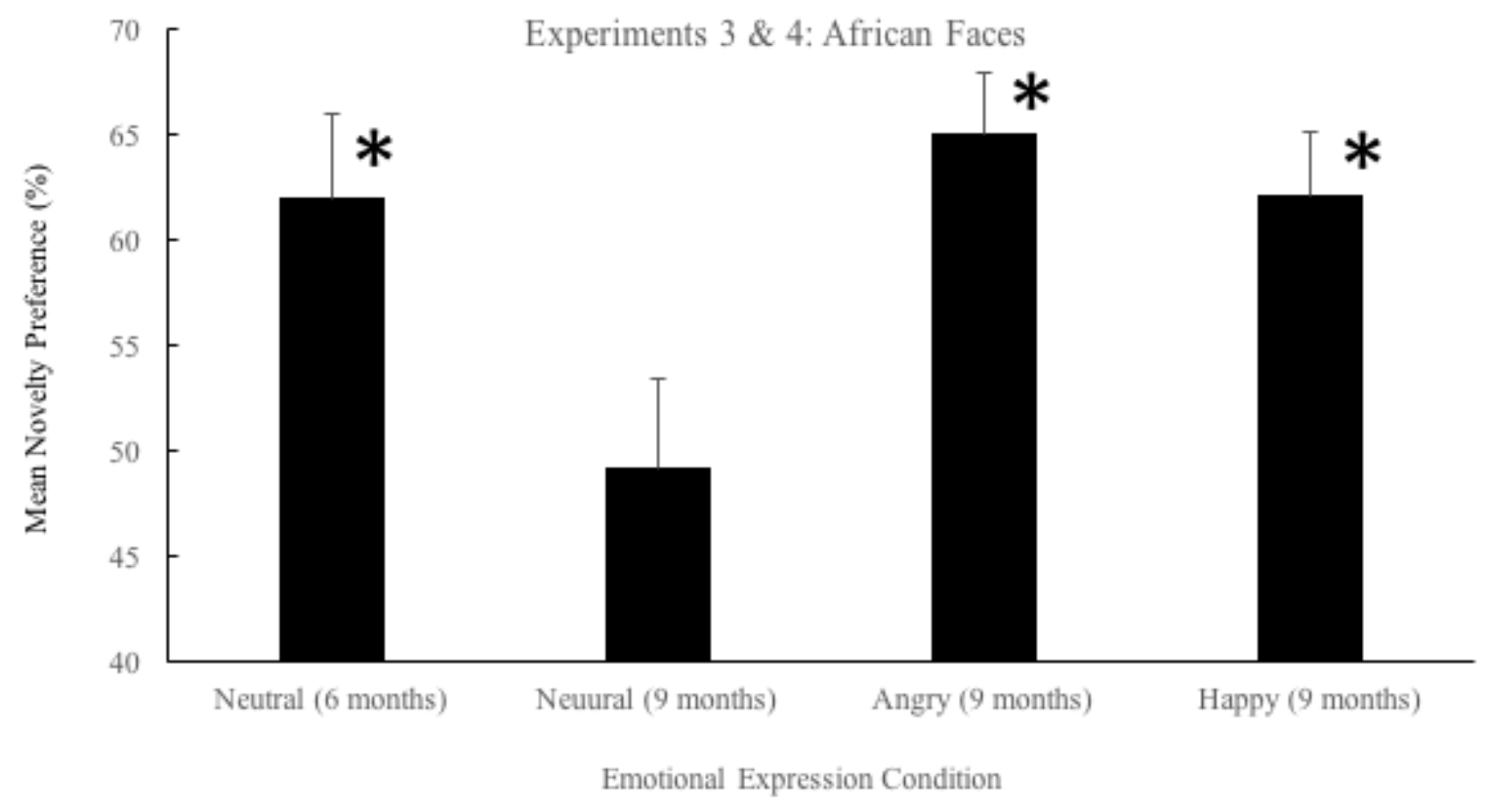

Figure 2. Mean novelty preference scores (in percentages) as a function of the emotional expression condition (neutral, angry, or happy) and the age of the participants (6 or 9 months) in Experiments 3 and 4. Error bars show standard errors of the mean. Scores that differed from chance $(50 \%)$ are marked with an asterisk. 